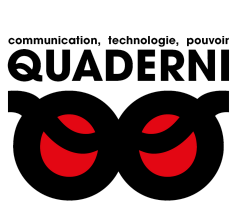

\title{
Quaderni
}

Communication, technologies, pouvoir

95 | Hiver 2017-2018

Logiques numériques des radicalisations

\section{Comment qualifier les relations entre les médias sociaux et les processus de radicalisation menant à la violence?}

Note de synthèse internationale

Séraphin Alava, Divina Frau-Meigs et Ghayda Hassan

\section{(2) OpenEdition \\ Journals}

Édition électronique

URL : https://journals.openedition.org/quaderni/1137

DOI : 10.4000/quaderni. 1137

ISSN : 2105-2956

Éditeur

Les éditions de la Maison des sciences de l'Homme

Édition imprimée

Date de publication : 5 février 2018

Pagination : 39-52

\section{Référence électronique}

Séraphin Alava, Divina Frau-Meigs et Ghayda Hassan, « Comment qualifier les relations entre les médias sociaux et les processus de radicalisation menant à la violence? », Quaderni [En ligne], 95 I Hiver 2017-2018, mis en ligne le 05 février 2020, consulté le 04 septembre 2021. URL : http:// journals.openedition.org/quaderni/1137; DOI : https://doi.org/10.4000/quaderni.1137 


\section{$D$ ossier}

\section{Comment qualifier les relations}

entre les médias sociaux et les processus de radicalisation menant à la violence? Note de synthèse internationale

\author{
Séraphin \\ Alava \\ Université de Toulouse 2 Jean Jaurès \\ Divina \\ Frau-Meigs \\ Université de Paris 3 \\ Ghayda \\ Hassan \\ Université de Québec \\ Montréal UQAM
}

Bien que la littérature grise soit très abondante (rapports, profils, enquêtes journalistiques approfondies...) et marquée par une forte augmentation de son volume au cours des dernières années (à partir de 2012), le domaine de la radicalisation des « jeunes » en ligne est encore insuffisamment étudié et théorisé. La plupart des recherches ont tendance soit à être anecdotiques (basées sur des études de cas isolés), soit peu abondantes en données empiriques (petits corpus, ensembles de données limitées, analyses ponctuelles...).

Dans cet article, nous portons attention à la radicalisation djihadiste en présentant les résultats les plus marquants sur les liens entre radicalisation et médias sociaux.

Cette étude ne montre pas de lien de causalité net entre la propagande en ligne et la radicalisation des jeunes. Le rôle précis d'Internet dans le processus de la radicalisation doit être exploré avec plus de soin. La recherche reconnaît l'efficacité de la propagande en ligne, mais Internet se rapproche davantage d'un environnement simplifiant pour la radicalisation plutôt qu'il ne ressort comme une force motrice. Les médias sociaux ne peuvent pas être examinés séparément des autres facteurs liés à des conditions hors ligne, même si les dimensions 'en ligne' et 'hors ligne' deviennent de plus en plus perméables. La socialisation hors ligne aux idéologies extrémistes tend à avoir lieu avant l'endoctrinement en ligne.

Cependant, une fois le point de non-retour franchi, les jeunes extrémistes trouvent de la matière dans les médias sociaux pour alimenter leur intérêt en devenant des proies faciles pour les stratégies de recrutement des groupes radicaux. 


\section{Les réseaux sociaux en accusation}

Les attentats dans le monde, les formes médiatiques de communication des groupes terroristes, la mise en évidence d'un espace numérique de diffusion des idées les plus extrêmes, tout cela a peu à peu fait émerger l'idée d'une responsabilité centrale de l'Internet et particulièrement des réseaux sociaux dans la croissance des actes radicaux. Les médias sociaux conduisent-ils les individus vulnérables à recourir à la violence ? Beaucoup d'hommes et de femmes politiques comme l'opinion publique le croient. L'Internet est alors présenté par certains comme un des vecteurs efficaces de radicalisation de notre jeunesse. La réaction de l'opinion publique ne tarde pas et les demandes de suppression ou de référencement des sites terroristes ou haineux accompagnent une demande croissante de surveillance des réseaux sociaux. La mise en place de campagnes actives des autorités nationales mais aussi des entreprises du Net en valorisation des contre-discours numériques donne le sentiment que la guerre contre le terrorisme se déploie aujourd'hui nettement dans le cyberespace. Mais que savons-nous vraiment sur les liens existants entre Internet et la propagation des discours et des actes violents? Que savons-nous sur l'impact des usages numériques des jeunes sur l'engagement radical? Partout dans le monde, les gouvernements et les entreprises d'Internet prennent des décisions sur la base d'hypothèses de causalités souvent non scientifiquement démontrées. L'action publique nécessite une politique construite sur la base des faits et des preuves, et non fondée sur des intuitions - ou menée par la panique et la peur. Pourtant le procès semble instruit : «L'Internet et les réseaux sociaux sont les complices du ter- rorisme et participent à l'embrigadement rapide de notre jeunesse. »

C'est dans ce contexte que l'UNESCO a lancé une étude intitulée « Les médias sociaux et la radicalisation de la jeunesse menant à l'extrémisme violent $»$. Ce travail de synthèse de la littérature scientifique mondiale a fourni une cartographie globale de la recherche (principalement entre 2012 et 2016) sur les rôles assumés par les médias sociaux dans les processus de radicalisation violente, en particulier lorsqu'ils affectent les « jeunes » et les femmes. La recherche répond à la conviction que l'Internet dans son ensemble est un vecteur actif de radicalisation violente qui facilite la prolifération des idéologies extrémistes violentes. En effet, de nombreuses recherches montrent que les groupes radicalisés et les recruteurs extrémistes sont bien répandus sur Internet. Il existe de plus en plus de connaissances sur la façon dont les terroristes utilisent le cyberespace. Il est en revanche moins évident de mesurer l'impact de cette diffusion des idées radicales. L'impact même des contre-mesures contribuant à promouvoir des alternatives pacifiques est encore moins connu. Bien qu'Internet puisse jouer (dans un sens profane) un rôle de facilitateur, l'existence d'un lien causal entre l'Internet et la radicalisation idéologique, violente ou la perpétration d'actes réels de violence extrémiste est loin d'être démontrée.

Cet article s'appuie sur un travail de synthèse réalisé durant plus d'une année sur la base d'un inventaire des recherches et d'un examen précis des méthodologies et des résultats de travaux internationaux. Il vise à répondre à la prévention de la radicalisation qui constitue un enjeu 
politico-social urgent et précise l'état actuel de nos connaissances pour mieux armer les décisions publiques et la compréhension individuelle du phénomène de radicalisation.

\section{Radicalisation, violence, terrorisme, de quoi parle-t-on ?}

Parce que les termes de « radicalisation », de « terrorisme » et de « guerre contre le terrorisme » sont au cœur d'une polémique de communication dans les médias traditionnels autant que dans les médias sociaux, l'utilisation de ces termes doit également être précisée par rapport aux hypothèses posées dans cet état de l'art. Nous avons choisi de combiner les termes et les spécifications sémantiques qui leur sont associées (Rousseau et al., 2016). En effet, les mots dénotés et leur étiquetage ne sont pas isolés de la sphère sociale qu'ils représentent ou dans laquelle ils sont insérés. En fait, ils sont révélateurs d'outils politiques puissants qui justifient et mobilisent l'action. Par conséquent, les définitions concernent des champs sémantiques hautement politisés, qui fluctuent avec les changements dans les priorités politiques.

Nos travaux tiennent compte des approches critiques de la sociologie de la radicalisation (Guibet \& Lafaye, 2016), qui soulignent les contradictions politiques, culturelles et sociales de la notion même de radicalisme. Le radicalisme a été utilisé de manière inappropriée et interchangeable avec des notions telles que l'intégrisme (qui se réfère généralement à une approche religieuse), l'endoctrinement (qui se réfère à un processus de contrôle mental), le djihad, l'extrémisme et le terrorisme, entre autres. Parce que le terme de radicalisation est abondamment employé et pris dans divers traits rhétoriques, il est essentiel de tirer parti de l'origine de ce terme, qui est lié au mot « racine », l'origine fondamentale d'une idée ou d'une cause.

La radicalisation, dans son sens épistémologique, se réfère à l'ancrage de sa connaissance, de ses opinions, de ses valeurs et de ses croyances pour déterminer son comportement. Dans ce sens du mot, les théories politiques révolutionnaires comme le marxisme, ou les mouvements sociaux comme les suffragettes, ou la lutte de Gandhi contre la colonisation britannique et les théories scientifiques révolutionnaires telles que la théorie de la relativité d'Einstein peuvent être qualifiés d'idées radicales. En outre, tout changement social tel que le féminisme radical, la fin de l'esclavage ou de la colonisation, etc. repose sur des positions radicalisées qui forment et soutiennent les transformations sociales. En ce sens, ils sont « extrémistes » lorsqu'ils sont considérés du point de vue d'un statu quo. Beaucoup de ces théories et mouvements ont considérablement contribué à l'amélioration des sociétés, des libertés et des droits individuels, avec ou sans violence. Comme le montre Moscovici (1976), de tels mouvements peuvent parfois être considérés comme la source de l'innovation et du changement social. Il est donc essentiel de distinguer la radicalisation des idées de la radicalisation des actions, et de distinguer la radicalisation vers l'extrémisme, la radicalisation violente (lorsque l'extrémisme devient violent) et celle des actes de violence extrémiste, y compris le terrorisme (comme forme particulière et logique de violence).

À l'heure actuelle, il n'y a pas de définition consensuelle de la radicalisation. Les auteurs 
s'y réfèrent de différentes façons. Suivant une approche multidisciplinaire et à plusieurs niveaux, ce rapport s'appuie sur trois définitions complémentaires de la radicalisation. La première définition émane de Farhad Khosrokhavar (2015) qui définit le concept de « radicalisation » religieuse à un micro-niveau, en mettant l'accent sur la personne. La radicalisation violente est analysée comme un engagement individuel et un processus d'endoctrinement dans des actions violentes, avec une attention particulière aux processus d'influence émotionnels et cognitifs. Selon Khosrokhavar, la radicalisation est un processus par lequel un individu ou un groupe vient prendre une forme d'action violente. Il est directement lié à une idéologie extrémiste qui conteste l'ordre établi au niveau politique, social ou culturel.

La deuxième définition est dérivée du sociologue allemand Wilhelm Heitmeyer $(1989$; 2002) qui a examiné les processus générant l'extrémisme de droite. Il a développé une théorie de la désintégration sociale en tenant compte des différents niveaux de l'écosystème d'un individu et de leur interrelation. Cela concerne les arguments socio-psychologiques de l'École de Francfort où la radicalisation violente est considérée comme le produit d'une combinaison d'expériences individuelles et de conditions sociales qui génèrent des griefs sociaux. Selon Heitmeyer (1989), «l'orientation extrémiste de droite se caractérise par des éléments ou des alternatives contre les modèles sociaux définis théoriquement, mais pratiquement est souvent basée sur des promesses non réalisées de démocratie politique, de possibilités individuelles de liberté et d'égalité» (Heitmeyer, 1989, p 164-76). Ainsi, à un niveau macro, la radicalisation violente de l'extrême droite peut être considérée comme une forme de guerre ou d'une guérilla, défiant des relations de pouvoir établies depuis longtemps et mettant en danger les libertés et la sécurité civile. Cette acception est restreinte puisqu'elle suppose que la radicalisation s'accompagne nécessairement d'actes de violence.

Sur la base du cadre écosystémique, Schmid (2013) utilise une troisième définition qui présente la radicalisation comme processus individuel ou collectif. Le terme émerge de la friction des relations intercommunautaires et il est associé à une situation de polarisation politique. Les pratiques de dialogue, de compromis et de tolérance entre les différents acteurs sont abandonnées, par au moins une des parties, en faveur d'une escalade de tactiques conflictuelles et violentes. Cette définition englobe également l'impact sérieux souvent négligé de la rupture dans le dialogue démocratique et l'engagement de la citoyenneté. Il a l'avantage de décrire comment les discours collectifs idéologiques, endoctrinants et collectifs sont construits là où les « autres » sont représentés radicalement différents de « nous ». Ce « nous » étant alors interprété comme abusé, menacé, les victimes doivent être défendues, tandis que l'« autre » est déshumanisé (par exemple, construit comme un mal, comme la catégorie « Kafir », « infidèles », utilisée par la radicalisation islamiste, ou « cochons, animaux » utilisés par les extrémistes de droite en référence à différents groupes) (Das, 1998). En conséquence, l'acte justifie l'exclusion, la persécution et la violence possible. Toutes ces définitions susmentionnées qui chacune pour leur part semble éloignée de la notion de terrorisme nous permettent de conceptualiser la radicalisation violente comme 
la coexistence dynamique et complémentaire d'au moins trois niveaux observables à la fois dans le monde réel et dans le cyberespace :

- La recherche de la signification fondamentale, de l'origine et du retour à une idéologie-racine ;

- La démarche d'embrigadement ou d'adoption par un groupe d'une forme violente d'expansion de ses propres idéologies ;

- La polarisation de l'espace social et la construction collective d'un idéal « nous » menacé contre eux, où les autres sont déshumanisés par un processus de bouc émissaire. Dans cette approche les liens entre théories du complot et radicalisation est à développer (Giry, 2015 ; Hall, 2006). L'approche conspirationniste étant présente dans la plupart des radicalisations violentes, mais bien plus large que la dérive violente et terroriste qui nous concerne.

Au sortir de cette revue de littérature conceptuelle nous aurions presque la sensation que tout est radicalisation, et ce mot perd du sens pour devenir un mot valise englobant des radicalisations « constructives » appuyée sur des faits (controverses scientifiques) et celles qui sont orientées vers la mort, la destruction. Nous pourrions laisser croire que la vision personnelle nihiliste d'un individu adorant la mort et le chaos est une radicalisation comparable à la pensée religieuse extrême visant la préparation du « royaume de sa foi ». Il n'existe donc pas aujourd'hui de pensée complexe et systémique permettant de faire de ce mot un objet scientifique. Nous avons donc choisi une méthode plus opérationnelle, s'appuyant sur la demande formulée par l'UNESCO qui consis- tait à nous demander de fournir une synthèse détaillée des recherches mondiales portant sur les liens ou sur les impacts entre les médias sociaux et les processus de radicalisation dans son assertion actuelle, c'est-à-dire le processus sous-jacent à des phénomènes extrémistes violents utilisant le terrorisme comme modalité d'action. Nous avons donc choisi de définir le mot « radicalisation » renvoyant aux 4 types de radicalisation suivants :

- Radicalisation politique. Nous incluons ici les phénomènes de radicalisation violente d'extrême droite, d'extrême gauche, nationaliste ou indépendantiste.

- Radicalisation religieuse. Nous incluons ici les actes de radicalisation violente qui prennent appui sur des idéologies religieuses, même si les religions sollicitées ne se reconnaissent pas dans ces actes. «Oui, le terrorisme existe, mais l'islam $n$ 'a rien à voir avec ce terrorisme », déclare le grand imam Ahmed el-Tayeb de l'université AlAzhar lors de la visite du pape François.

- Radicalisation sociale. Nous incluons dans cette typologie l'ensemble des radicalisations menant à la violence de groupes armés ou non qui rejettent l'autre à travers des discriminations de quartiers, de sports, de genres, de bandes ou de groupes armés.

\section{- Radicalisation communautaire ou ethnique.}

Nous incluons dans ce type toutes les radicalisations violentes ethniques ou communautaires.

Ces quatre typologies ne relèvent pas bien sûr d'observables distincts mais ce sont des idéaux types qui peuvent se mixer ou s'hybrider. Nous 
avons conscience dans cette opérationnalisation que nous perdons en qualité théorique mais comme le champ est aujourd'hui objet de controverses nous avons choisi pour répondre à une demande sociale de rester dans des idéaux types opérationnalisables.

\section{Méthodologie de notre synthèse. Revue de littérature internationale et modalités de sélection}

Afin de respecter les objectifs d'une revue de littérature internationale, nous avons développé une solide méthodologie d'évaluation en nous appuyant sur les méthodes Campbell (Boruch, 2004). Nous avons peaufiné les détails de chaque étape en fonction de la nature et des objectifs spécifiques de notre travail à partir du modèle de Cochrane et PRISMA (https://www.campbellcollaboration.org). Chaque étape d'examen est décrite ci-dessous, ainsi que les résultats de la recherche d'évaluation. Ces étapes sont des étapes clés pensées en interaction dans un processus itératif continu.

Notre travail de recension, de sélection, de lecture et de synthèse a respecté les étapes suivantes :

- Étape 1. Formulation de la question et stabilisation des définitions : " Quels sont les rôles des médias sociaux dans le processus de radicalisation violente et dans quelle mesure les incidents de prévention / intervention jouent-ils ce rôle? » Dans ce travail, nous avons exclu les formes structurées de la guérilla ou de la guerre civile, des acteurs révolutionnaires avec leurs formes violentes, des formes violentes structurées par des organisations illégales (mafia, gangs). Notre travail exclut également les formes radicales de pensée (antisystème, populisme, conspiration). En effet ce choix n'implique pas que les modes de pensée conspirationniste ne participent pas au processus de radicalisation comme d'ailleurs des éléments des discours de rupture comme les notions de discriminations religieuses ou de ghettoïsation mais nous ne souhaitions pas trop élargir notre champ et les liens entre complotisme et radicalisation n'ont pas été démontrés même s'il nous apparaît que le complotisme participe à la construction d'une distinction « eux et nous » qui est une des thématiques de l'embrigadement.

- Étape 2. Définir les critères d'inclusion / d'exclusion : les critères que nous avons utilisés pour établir les éléments de preuves admissibles sont fondés sur un examen contradictoire des éléments et s'appuient sur la capacité à formuler des recommandations nettes. Puisque la recherche du lien entre Internet et la radicalisation violente est relativement nouvelle, nous sommes intéressés par l'approche " Best Available Evidence », afin de valoriser les résultats les plus évidents s'appuyant sur des données explicites et objectives. Cette technique utilisée en criminologie va appuyer ses analyses d'abord sur les faits établis en essayant de ne pas privilégier des faits intéressants mais non établis.

- Étape 3. Recherche de la littérature : chaque équipe nationale a développé la stratégie de recherche et l'a adaptée à ses bases littéraires scientifiques et grises (articles journalistiques ou publicitaires disponibles pour les scientifiques). Nous avons ensuite effectué des recherches systématiques sur les bases de données les plus utilisées et les plus connues internationalement. 
- Étape 4. Sélectionner les études à inclure dans l'examen : premièrement, les membres de chaque équipe de recherche du pays ont examiné les résumés, afin d'éliminer les études clairement non éligibles. Deuxièmement, ils ont examiné les documents complets pour le dépistage final de l'éligibilité en utilisant une échelle de 1 (exclusion définitive) à 5 (inclusion définitive). Les désaccords ont été discutés et les décisions ont été obtenues par consensus.

- Étape 5. Recueillir des informations provenant des études : les données et les informations de chaque étude sélectionnée ont été examinées pour la première. Les informations et les données ont ensuite été extraites à l'aide de feuilles de codage pour l'extraction de données.

- Étape 6. Analyser et intégrer les résultats de la recherche : nous avons effectué plusieurs agrégations de données parallèles selon : a) les différents concepts issus du processus d'examen; b) le type d'étude ; c) la zone géographique d'étude ; d) les études axées sur la prévention ou l'intervention. Les membres de l'équipe de recherche ont procédé à l'extraction et à la synthèse des données.

- Étape 7. Interpréter les éléments de preuve : dans cette étape, nous avons synthétisé les preuves accumulées en particulier : a) les différents thèmes qui ont émergé de la littérature ; b) le degré de confiance dans chaque conclusion (en termes d'information disponible et de robustesse des études et des rapports) ; c) la généralisation et l'applicabilité des conclusions ; d) les limites des connaissances existantes.

\section{Cartographies des recherches et des cher- cheurs}

4517 articles présentent des données quantitatives sur la base d'un corpus constitué des publications portant sur les questions de terrorismes et de radicalisation. 1956 articles de cette base ont effectué des croisements avec les thèmes numériques (Internet, médias sociaux). Seulement 594 articles sont retenus pour la qualité des travaux et la qualité des informations utilisables. Concernant la répartition des langues de travail, l'anglais domine mais le français et l'allemand sont très bien représentés (18 et 10\%), et seulement $6 \%$ en arabe. En revanche, si on braque le projecteur sur les auteurs ( $1^{\mathrm{er}}$ nom), les auteurs arabophones sont $20,5 \%$ contre $23,3 \%$ pour les Américains et 42,3\% pour les Européens.

\section{État de la recherche dans le domaine des liens entre médias sociaux et radicalisation violente et résultats}

Malgré la prépondérance des discours politiques ou médiatiques précisant ou dénonçant un lien fort entre le déploiement des médias sociaux et l'émergence des formes autochtones de radicalisation, notre travail de synthèse révèle que peu de recherches ont porté sur le rôle ou sur l'efficacité de l'utilisation des médias sociaux dans la radicalisation violente. Bien qu'un grand nombre d'articles traitent des stratégies électroniques et des utilisations de l'Internet et des médias sociaux en ligne pour le recrutement terroriste, il existe très peu d'études empiriques qui décrivent et examinent les effets réels de ces stratégies sur les jeunes (Ernst-Vintila, 2015). De même, les aspects liés au genre sont rarement 
examinés. Certes les domaines de recherches sont très récents et il est parfois difficile de démêler les différents aspects sous-jacents derrière le mot-clé « Internet » dans la littérature Web et les médias sociaux avant 2005. Notons qu'en dépit d'une recherche plus ciblée à compter de 2010, les médias sociaux étaient encore inclus dans la métaphore du « réseau » (Goede, 2012), qui était utilisée pour englober l'Internet entier, y compris les applications Web et les plates-formes dérivées. La métaphore du réseau comporte une certaine quantité de valeurs, telles que la connectivité transfrontalière, et une plate-forme qui rend le monde susceptible d'intervention. Mais il est également de plus en plus identifié au développement d'un risque technologique, entraînant un danger pour la propagation rapide des virus, des rumeurs et de la haine. En ce qui concerne la radicalisation, l'extrémisme et le terrorisme, le réseau n'est pas seulement une partie du problème, mais également une partie de la solution.

Aujourd'hui nous constatons que l'état de l'art indique qu'il n'y a pas de travaux ayant établi un lien causal entre réseaux sociaux et radicalisation, ce qui ne veut pas dire que l'Internet n'est pas un levier pour la radicalisation, seulement que cette affirmation n'est ni prouvée ni réfutée. Les utilisations actuelles des médias sociaux et les questions de réglementation de l'information en ligne méritent des recherches pour savoir si elles sont effectivement utilisées comme contributeur à la radicalisation des idées et de développement de mentalités extrémistes violentes et d'actions possibles, en particulier chez les jeunes hommes et les jeunes femmes.

La plupart des recherches entre 2005 et 2011 tendent à se concentrer sur les utilisations positives de l'Internet et des médias sociaux par les " jeunes », même dans le cadre du programme «Internet plus sûr » de l'UE en 1999. Quelques études examinent les utilisations négatives des adultes (principalement en relation avec la pédophilie, la pornographie et la violence causée par la cyberviolence ou le harcèlement ciblé (Livingstone et al., 2011 ; Blaya, Alava, 2012). Les stratégies individuelles plutôt que collectives sont également l'objet principal de l'attention, et très peu d'études portent sur l'utilisation extrémiste (politique ou religieuse) du cyberespace. Dans cette période, les activistes sur la toile développant des idées radicales ne sont pas encore clairement identifiés comme des groupes politiques, mais plutôt comme une partie des sous-cultures en ligne. Cette indexation peut avoir entrâné une sous-estimation de la rapidité de leur transformation en réseau et de leur puissance idéologique.

Depuis 2012, en partie en raison du « printemps arabe » et du pouvoir de convocation des médias sociaux, certaines recherches ont mis en évidence la similitude des réseaux sociaux et des groupes terroristes dans leur fonction de réseaux dans la mesure où ils sont décentralisés, omniprésents et mobiles (Conway 2007 ; Schils \& Laffineur, 2013). Avec Internet et les médias sociaux, le public passe de groupe de spectateurs passifs à des agents actifs qui « recueillent des informations par eux-mêmes, plutôt que d'attendre que les médias les filtrent et les délivrent » (Seib, 2006, p. 78). La socialisation s'étend à la production et au partage d'informations au sein de ces réseaux en ligne. Les fonctionnalités d'Internet ont entraîné une transformation des tactiques des 
groupes extrémistes. Ces groupes se sont adaptés au potentiel dématérialisé du web. Internet offre des informations en plus grand volume et à plus grande vitesse, ainsi que dans divers formats, notamment des vidéos pour une communication visuelle et fondée sur l'émotion, la communication bidirectionnelle d'interactivité, les liens horizontaux et autres (Ney, 2015). Il est également décentralisé et ouvert au contrôle individuel (Tsfati \& Weimann, 2002, Weimann, 2006). Il offre la possibilité de rechercher ou de publier des informations sous un relatif anonymat et loin de la surveillance ou du contrôle du gouvernement, en particulier lorsque les pays ayant des niveaux élevés de protection de la liberté d'expression ont peu de censure sur le contenu Internet. En outre, les informations peuvent être affichées sur les réseaux locaux tout en ciblant un public mondial à travers le temps et l'espace, atteignant leur but n'importe où et n'importe quand (Conway, 2007; Schils \& Laffineur, 2013). Les plates-formes de médias sociaux offrent des avantages significatifs aux groupes extrémistes qui, autrement, auraient pu rester marginaux en termes de moyens de communication.

Des recherches récentes ont porté sur 1'utilisation croissante des sites de réseautage social des extrémistes violents, tels que Facebook et Twitter, avec des incursions supplémentaires dans YouTube - utilisés pour la propagande (le web comme « théâtre de terreur », Weimann, 2008), le recrutement (Weimann, 2006) et la collecte de fonds. La littérature est principalement constituée de rapports descriptifs de la littérature grise. Certaines contributions intéressantes incluent le rapport intitulé \#Greenbirds : mesurer l'importance et l'influence dans les réseaux de combattants étrangers syriens qui documentent l'utilisation des médias sociaux pour le recrutement décentralisé et le ciblage de jeunes publics (ICSR, 2013).

Le rapport « Tringer Jihad » réalisé par la Fondation Quilliam (2014) décompose chaque réseau social majeur et analyse ses caractéristiques, les compare et les oppose dans leurs rôles respectifs, ainsi que les contrastes avec les sites Web et les salles de discussion. Il est important de noter que les rapports mentionnés ci-dessus se concentrent uniquement sur la radicalisation violente islamiste et n'examinent pas le spectre de toutes les autres formes et groupes de radicalisation violente. L'information qu'ils fournissent n'est donc pas nécessairement généralisable à d'autres groupes. En effet même si la presse semble construire des ponts entre radicalisation djihadiste et radicalisation d'extrême droite peu ou pas d'études scientifiques permettent de prouver cette liaison.

\section{Tchats et forums}

Les tchats et forums peuvent être intégrés dans la plupart des médias basés sur Internet. Les rapports qui ont examiné l'utilisation des salles de discussion par des groupes extrémistes violents, les décrivent comme un espace où « les jeunes à risque » sans exposition antérieure pourraient rencontrer des récits religieux radicalisants (Hussain, Saltman 2014 ; Shah, 2012). Cela s'inscrit dans le cadre de l'apport de Sageman (2004) sur le rôle des forums de discussion, en fonction de sa distinction entre les sites Web en tant que sources d'informations passives et les salles de discussion comme sources d'interactions actives. Selon Sageman, le réseautage est facilité par les 
forums de discussion parce qu'ils développent la communication entre les adeptes des mêmes idées (expériences, idées, valeurs), renforcent les relations interpersonnelles et fournissent des informations sur les actions (tactiques, objectifs, tutoriels).

\section{Facebook}

Bien que de nombreux groupes extrémistes soient idéologiquement et stratégiquement antiFacebook, certains conservent et développent une forte présence sur cette plate-forme, soit directement, soit par l'intermédiaire de supporters (Hussain, Saltman 2014). Cependant, Facebook ne semble pas être utilisé pour le recrutement ou la planification directe, peut-être parce qu'il dispose de mécanismes de suivi et peut lier les utilisateurs avec des lieux réels et des horaires spécifiques. Au lieu de cela, Facebook est plus souvent utilisé par les extrémistes comme un lieu décentralisé de diffusion des ressources d'informations et de vidéos ou comme un espace de recrutement direct (Hussain, Saltman 2014 ; Shah, 2012). Il est utilisé pour attirer des membres dans l'idée que les jeunes sympathisants puissent partager des informations et des images afin de créer des groupes Facebook de manière décentralisée.

\section{Twitter}

Les sites de microblogging comme Twitter présentent plus d'avantages pour les groupes extrémistes, car la traçabilité de l'identité et la source des tweets sont plus difficiles à atteindre, augmentant ainsi le potentiel de communication pour les recruteurs (Crettiez, 2011; Hussain,
Saltman 2014; Menkhaus, 2014). De plus, via Twitter, les extrémistes peuvent facilement commenter publiquement des événements internationaux, en plusieurs langues, ce qui permet aux militants d'être réactifs lors du montage de campagnes virales de désinformation (Hussain, Saltman 2014 ).

\section{YouTube et autres plates-formes vidéo}

YouTube reste la plate-forme de partage de vidéos de choix malgré la concurrence accrue d'autres plates-formes, telles que Dailymotion, Vimeo et autres. Son l'avantage : l'identité des personnes affichant du contenu est difficilement repérable et elle offre la possibilité aux utilisateurs de générer des commentaires et de partager des contenus (Quilliam, 2016). Les trois principales raisons qui motivent les extrémistes violents islamistes à générer des messages vidéo sont l'éloge des martyrs, l'encouragement aux attentats suicides et la propagande en faveur de l'idéologie extrémiste (Conway \& McInerney 2008; Hussain, Saltman 2014, Hecker, 2015). Le rapport Quilliam (2016) publie une analyse complète du contenu des vidéos extrémistes violentes islamistes publiées sur YouTube. Plusieurs chercheurs ont mené des analyses de contenu sur les discours extrémistes de YouTube et Facebook ainsi que sur les vidéos pour identifier les caractéristiques de production les plus utilisées, y compris leurs modes opératoires et leurs effets prévus (Salem, Reid \& Chen, 2008; Tomomi, 2013). Les études axées sur la stratégie rhétorique des groupes extrémistes montrent l'utilisation multiple des ressources en ligne par des groupes extrémistes. C'est-à-dire qu'ils produisent une « séduction numérique » en utilisant des motifs visuels qui sont familiers 
« aux jeunes » en ligne (Vergani, 2014). Ces vidéos fournissent des messages multimédias riches multilingues combinant des indices non verbaux et des images vives d'événements qui peuvent provoquer des réactions psychologiques et émotionnelles, ainsi que des réactions violentes (Salem, Reid \& Chen, 2008). Les terroristes filment leurs attaques sur les vidéos et les diffusent par Internet, en communiquant une image d'efficacité et de réussite.

\section{Conclusion}

La radicalisation des jeunes en ligne n'a pas encore attiré suffisamment d'études pour que la recherche soit crédible dans ses conclusions et ses recommandations. Il n'existe actuellement qu'une petite quantité de données qualitatives sur le sujet, contrairement à la littérature sur la responsabilisation des jeunes en matière d'usage sécurisé de l'Internet. Bien que la littérature grise (rapports, profils, enquêtes journalistiques approfondies) sur la radicalisation violente et le terrorisme se soit développée au cours des dernières années (à partir de 2012), le domaine académique est encore insuffisamment étudié et théorisé. En outre, plusieurs études subissent des limites méthodologiques importantes telles que des études de cas anecdotiques (basées sur des études de cas isolés), soit peu abondantes en données empiriques (des petits corpus, ensembles de données limitées, analyses ponctuelles...). Il y a aujourd'hui beaucoup d'analyses indirectes de cas (procès, presse) et peu d'analyses qualitatives directes poussées.

Actuellement, il existe des preuves d'affinités électives entre l'exposition à la propagande extrémiste et le recrutement, la manifestation d'attitudes extrémistes et un risque accru de radicalisation violente chez les jeunes, en particulier dans le cas de groupes d'extrême droite. Cependant, les rôles et les processus exacts par lesquels l'Internet et les médias sociaux contribuent au processus de radicalisation doivent encore être explorés. Aussi, il n'existe pas de preuves suffisantes pour conclure qu'il existe un lien de causalité entre la propagande extrémiste en ligne ou le recrutement par Internet et les médias sociaux et la radicalisation violente des jeunes. L'Internet est devenu un espace de rencontre entre des individus perdus ou en quêtes de sens et des discours structurés s'appuyant sur des discours conspirationnistes et extrémistes.

La synthèse de la littérature montre que, au mieux, les médias sociaux constituent un environnement facilitant plutôt qu' une force motrice pour la radicalisation violente ou la véritable commission de la violence. L'état de l'art montre qu'il n'existe aucune preuve tangible que l'influence des médias sociaux puisse agir indépendamment d'autres facteurs hors ligne, bien que les dimensions en ligne et hors ligne deviennent de plus en plus poreuses. En ce sens, l'Internet et les médias sociaux peuvent servir de renfort parce que les jeunes extrémistes peuvent alors chercher et trouver activement de la matière pour nourrir leurs intérêts et devenir des proies faciles face aux stratégies de recrutement des groupes radicaux. 


\section{$R \cdot E ́ F \cdot E ́ R \cdot E \cdot N \cdot C \cdot E \cdot S$}

ALAVA S., FRAU-MEIGS D., HASSAN G. (2017). Youth and violent extremism on social media : Mapping the research, With the collaboration of Hasna Hussein and Yuanyuan Wei. Published in 2017 by the United Nations Educational, Scientific and Cultural Organization 7. http://unesdoc.unesco.org/ images/0026/002603/260382e.pdf

BLAYA C., ALAVA S., (2012). Risks and safety for children on the internet: the French report, LSE, London: EU Kids Online.

BORUCH R., SOYDAN H., DE MOYA D., \& Campbell Collaboration Steering Committee. (2004). The Campbell collaboration. Brief Treatment and Crisis Intervention, 4(3), 277-287.

CONWAY M. (2009). Le cyber-terrorisme.: Le discours des médias américains et ses impacts. Cités, 39(3), 81.

CONWAY M., MCINERNEY L. (2008). Jihadi Video and Auto-radicalization: Evidence from an Exploratory YouTube Study Intelligence and Security Informatics, Proceedings (Vol. 5376, p. 108-118).

CRETTIEZ X. (2011). « High risk activism » : essai sur le processus de radicalisation violente (première partie). Pôle Sud, (34), 45-60.

DAS V. (1998), “ Specificities: Official Narratives, Rumor, and the Social Production of Hate. Social Identities", Journal for the Study of Race, Nation and Culture, vol.4, $\mathrm{n}^{\circ}$, p. 109-130.

ERNST-VINTILAA. (2015). Mentalité conspirationniste, socialisation, radicalisation. Réflexion théorique et illustration empirique. Psihologia Socială, nr. 36 (II)/2015

GIRY J. (2015), « Le conspirationnisme, Arché- ologie et morphologie d'un mythe politique moderne », Diogène, n`249-250, p. 40-50.

GOEDE de M., SIMON S. (2012). Governing Future Radicals in Europe. Antipode, 14 septembre 2012

GUIBET L., C. (2016). Approche critique des sociologies de la radicalisation. Forum de la $D A P$ « Radicalisation violente, engagement et désengagement », Octobre 2016.

HALL J. A. (2006), "Aligning Darkness with Conspiracy Theory: The Discursive Effect of African American Interest in Gary Webb « Dark Alliance »", The Howard Journal of Communication, vol.17, n³, pp. 205-222.

HARAS R., NIKITA M. (2015). Caliphettes: Women and the Appeal of Islamic State. Quilliam Foundation. https://www.quilliamfoundation. org/wp/wp-content/uploads/publications/free/ caliphettes-women-and-the-appeal-of-is.pdf.

HECKER M., «Web social et djihadisme : du diagnostic aux remèdes ", Focus stratégique, $n^{\circ} 57$, juin 2015 .

HEITMEYER W. (2002). Gruppenbezogene Menschenfeindlichkeit. Die theoretische Konzeption und erste empirische Ergebnisse (Group-focused Enmity. Theoretical conception and first empirical results). In W. Heitmeyer (Ed.), Deutsche Zustände, Folge 1 (German states, Vol. 1) (pp. 15-36). Frankfurt a.M.: Suhrkamp. HUSSAIN G., SALTMAN E. M. (2014) Jihad Trending: A Comprehensive Analysis of Online Extremism and How to Counter it Quilliam, May 2014. Quilliam Foundation.

KHOSROKHAVAR F. (2015). Radicalisation. Paris, Les Éditions de la MSH.

LIVINGSTONE S., HADDON L., GÖRZIG A., ÓLAFSSON K. (2011) Risks and safety on the internet: The perspective of European children: 
Full findings. http://eprints.lse.ac.uk/33731/

MENKHAUS K. (2014). Al-Shabaab and Social Media: A Double-Edged Sword. https://www. questia.com/library/p436518/the-brown-journalof-world-affairs.

NEY J.-P. (2015). Pourquoi ils font le Djihad. Enquête sur la \#générationmerah. Artège.

ROUSSEAU C. \& al. (2016). Le défi du vivre ensemble : Les déterminants individuels et sociaux du soutien à la radicalisation violente des collégiens et collégiennes au Québec : Rapport de recherche. SHERPA, Institut Universitaire en regard aux communautés culturelles du CIUSSS Centre-Ouest-de-l'Île-de-Montréal.

SAGEMAN M. (2004). Understanding terror networks. Philadelphia: University of Pennsylvania Press.

SAGEMAN M. (2008). A strategy for fighting international Islamist terrorists. Annals of the American Academy of Political and Social Science, 618(1), 223-231.

SALEM A., REID E., CHEN H. (2006). Content analysis of jihadi extremist groups' videos Intelligence and Security Informatics, Proceedings (Vol. 3975, pp. 615-620).

SALEM A., REID E., CHEN H. (2008). Multimedia content coding and analysis: Unraveling the content of Jihadi extremist groups' videos. Studies in Conflict \& Terrorism, 31(7), 605-626. SCHILS N., LAFFINEUR J. (2013). Comprendre et expliquer le rôle des réseaux sociaux dans la formation de l'extrémisme violent. Belgique : université de Gand et université catholique de Louvain, BELSPO.

SCHMID Alex P. (2013). Radicalisation, DeRadicalisation, Counter-Radicalisation: A Conceptual Discussion and Literature Review. ICCT Research Paper, March 2013.
SEIB P. (2008). Terrorism and the Al Jazeera Effect. Conference Papers - International Studies Association, 1.

SHAH M., "Online social networks: The overt and covert communication channels for terrorists and beyond", Homeland Security (HST) 2012 IEEE Conference on Technologies for, p. 574-579, 2012.

TOMOMI, Yi. (2013). Xenophobia in Action: Ultranationalism, Hate Speech, and the Internet in Japan. Radical History Review (117), 98-118. doi: 10.1215/01636545-2210617.

TSFATI Y., WEIMANN G. (2002). Www. terrorism.com: Terror on the Internet. Studies in Conflict and Terrorism, 25(5), 317-332.

VERGANI M. (2014). Neojihadism and Muslim, ÄiChristian Relations in the Mindanao Resistance Movement: A Study of Facebook Digital Narratives. Islam \& Christian-Muslim Relations, 25(3), 357-372.

WEIMANN G. (2006). Terrorist dot com: Using the internet for terrorist recruitment and mobilization. The making of a terrorist, 1, 53-65.

WEIMANN G. (2008). The psychology of mass-mediated terrorism. American Behavioral Scientist, 52(1), 69-86.

WINTER, C. (2015). 'Documenting the Virtual Caliphate', Quilliam Foundation, October 2015, http://www.quilliaminternational.com/wp-content/uploads/2015/10/FINAL-documenting-thevirtual-caliphate.pdf. 
$R \cdot E ́ \cdot S \cdot U \cdot M \cdot E ́$

Les médias sociaux conduisent-ils les individus vulnérables à recourir à la violence ? Beaucoup de gens croient que c'est le cas. Et les États répondent par la censure en ligne, la surveillance et le contre-discours. Mais que savons-nous vraiment des liens entre Internet et violence, et que savons-nous de l'impact des réseaux sociaux sur les phénomènes de radicalisation? Partout dans le monde, les gouvernements et les sociétés Internet prennent des décisions sur la base de suppositions sur les causes et les remèdes face au terrorisme. Le défi sociétal est grand de comprendre ces liens. La nécessité d'une politique fondée sur des faits et des preuves, et non fondée sur des intuitions - ou poussée par la panique et la peur - est essentielle. C'est dans ce contexte que l'UNESCO a commandé l'étude intitulée Médias sociaux et radicalisation de la jeunesse à l'ère numérique. Ce travail fournit une cartographie globale de la recherche sur les rôles supposés des médias sociaux dans les processus de radicalisation violents, particulièrement en ce qui concerne les jeunes et les femmes.

\begin{abstract}
Does social media lead vulnerable individuals to resort to violence? Many people believe it does. And they respond with online censorship, surveillance and counter-speech. But what do we really know about the Internet as a cause, and what do we know about the impact of these reactions? All over the world, governments and Internet companies are making decisions on the basis of assumptions about the causes and remedies to violent attacks. The challenge is for analysis and
\end{abstract}

responses to be firmly grounded. The need is for policy that is constructed on the basis of facts and evidence, and not founded on hunches - or driven by panic and fearmongering. It is in this context that UNESCO has commissioned the study titled Social Media and Radicalization of Youth in the Digital Age. This work provides a global mapping of research into the assumed roles played by social media in violent radicalization processes, especially as they affect youth and women. 